\title{
On polygons enclosing point sets II
}

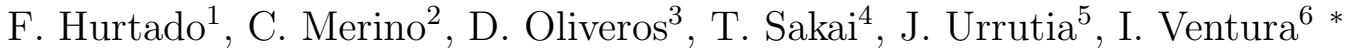 \\ 1 Departament de Matemàtica Aplicada II, Universitat Politècnica de Catalunya. \\ ${ }^{2}$ Instituto de Matemáticas, Universidad Nacional Autónoma de México, México D.F. México. \\ 3 Instituto de Matemáticas, Universidad Nacional Autónoma de México, México D.F. México. \\ 4 Research Institute of Educational Development, Tokai University, 2-28-4 Tomigaya, Shibuya- \\ ku, Tokyo 151-8677, Japan \\ 5 Instituto de Matemáticas, Universidad Nacional Autónoma de México, México D.F. México. \\ ${ }^{6}$ Departmento de Matemáticas, Universidad de Huelva, Huelva, España.
}

\begin{abstract}
Let $R$ and $B$ be disjoint point sets such that $R \cup B$ is in general position. We say that $B$ encloses by $R$ if there is a simple polygon $P$ with vertex set $B$ such that all the elements in $R$ belong to the interior of $P$.

In this paper we prove that if the vertices of the convex hull of $R \cup B$ belong to $B$, and $|R| \leq|\operatorname{Conv}(B)|-1$ then $B$ encloses $R$. The bound is tight. This improves on results of a previous paper in which it was proved that if $|R| \leq 56|\operatorname{Conv}(B)|$ then $B$ encloses $R$. To obtain our result we prove the next result which is interesting on its own right: Let $P$ be a convex polygon with $n$ vertices $p_{1}, \ldots, p_{n}$ and $S$ a set of $m$ points contained in the interior of $P$, $m \leq n-1$. Then there is a convex decomposition $\left\{P_{1}, \ldots, P_{n}\right\}$ of $P$ such that all points from $S$ lie on the boundaries of $P_{1}, \ldots, P_{n}$, and each $P_{i}$ contains a whole edge of $P$ on its boundary.
\end{abstract}

Key words. Enclosing polygon, red blue point sets.

\section{Introduction}

Let $S$ be a set of $n$ points in the plane in general position. A polygonization of $S$ is a simple polygon such that its vertex set is $S$. Finding an upper and lower bounds on the number of polygonizations any point set admits is a problem that has been receiving much attention since it was posed in 1979 by Akl [2] and 1980 by Newborn and Moser [13]. In [2] a lower bound of $2.27^{n}$ was proved. An upper bound of the form $c^{n}$ for some constant $c$ (ignoring polynomial terms) was conjectured by Newborn and Moser [13]. This was proved in 1982 by Ajtai, Chvátal, Newborn, and Szemerédi [1], who proved that there are at most $10^{13 n}$ crossing-free graphs on $n$ points in a paper that had strong influence in the latter theory of geometric graphs [3]. This bound has been improved in several papers, and most recently for polygonizations to $86.81^{n}$ by Sharir and Welzl [15].

Any simple polygon $P$ defines two open regions on the plane, a bounded one called the interior of $P$ and an unbounded region called the exterior of $P$. The area of $P$ is the

\footnotetext{
* F. Hurtado partially supported by projects MEC MTM2006-01267 and DURSI 2005SGR00692. C. Merino supported by CONACYT of Mexico, Proyecto 43098. J. Urrutia supported by CONACYT of Mexico, Proyecto SEP-2004-Co1-45876, and MCYT BFM2003-04062. I. Ventura partially supported by Project MCYT BFM2003-04062.
} 
area of the region bounded by $P$. Problems of finding polygonizations of point sets $S$ that maximize or minimize some parameters of the polygonization have also been studied. S.

Fekete [9] considers the problem of finding polygonizations of point sets $S$ that minimize or maximize the enclosed area. He proves that finding such polygonizations is $\mathrm{NP}$ complete. It is worth mentioning here that a tool used by Fekete, is Pick's Theorem, a classic result on polygonizations that for polygons with vertices on the integer lattice, establishes an elegant relation between the area of the polygons and the number of lattice points on the boundary and in the interior of such polygons $[14,4]$. The problem of finding polygonizations of point sets that minimize the perimeter is the famous Euclidean Travelling Salesman problem, and it is well known that this problem is NP-hard [11].

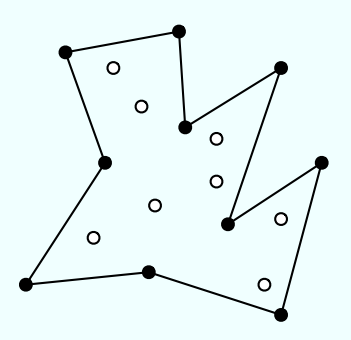

Fig. 1. The polygon through the points represented by small solid points encloses all of the points represented by small empty circles.

We say that a polygon $P$ encloses a point set $S$ if all the elements of $S$ belong to the interior of $P$; see Figure 1. Let $R$ and $B$ be disjoint point sets on the plane such that $S=R \cup B$ is in general position. The elements of $R$ (respectively $B$ ) will be called the red points of $S$ (respectively the blue points of $S$ ). A polygonization of $B$ will be called a blue polygonization. The problem of finding a blue polygonization that encloses as many red points as possible was studied in [5].

Since any red element of $S$ that is enclosed by a blue polygonization must belong to the interior of the convex hull $\operatorname{Conv}(B)$ of $B$, in what follows we will assume that all the elements of $R$ belong to the interior of $\operatorname{Conv}(B)$. Under this assumption, it is proved in [5] that there always exists a blue polygonization that encloses at least half of the elements of $R$. Moreover this bound is asymptotically tight. It is also proved that there always exists a polygon that covers at least half of the area of the convex hull of $S$.

Let $k$ denote the number of vertices on $\operatorname{Conv}(B)$, and $i$ the number of elements of $B$ in the interior of $\operatorname{Conv}(B), i+k=n$. In [5] it was also proved that if $|R| \leq 56 k$, then there always exists a blue polygonization that encloses $R$. It is easy to see that there are red point sets contained in $\operatorname{Conv}(B)$ with $k$ elements such that the whole of $R$ cannot be enclosed by any blue polygonization; simply let $R$ have an element close enough to the midpoint of each of the $k$ edges of $\operatorname{Conv}(B)$, and make sure that $B$ has at least one point in the interior of $\operatorname{Conv}(B)$ as shown in Figure 2.

Our main goal in this paper is to show that if $R$ has at most $k-1$ points, then there always exists a blue polygonization that encloses $R$. The main tool used here is a partitioning lemma that we consider to be of interest on its own, asserting the following: Let $P$ be a convex polygon with $n$ vertices, and $S$ a point set contained in $P$ with at most $n-1$ elements. Then there is a set of $n$ convex polygons $P_{1}, \ldots, P_{n}$ with disjoint interiors 


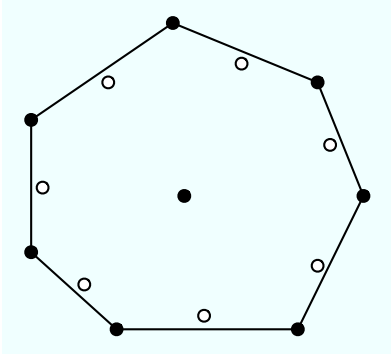

Fig. 2. The blue points are represented by small solid circles, the red ones by small empty circles.

such that the elements of $S$ belong to the boundaries of $P_{1}, \ldots, P_{n}$, each $P_{i}$ contains on its boundary exactly one edge of $P$, and $P_{1} \cup \ldots \cup P_{n}=P$. See Figure 3 .
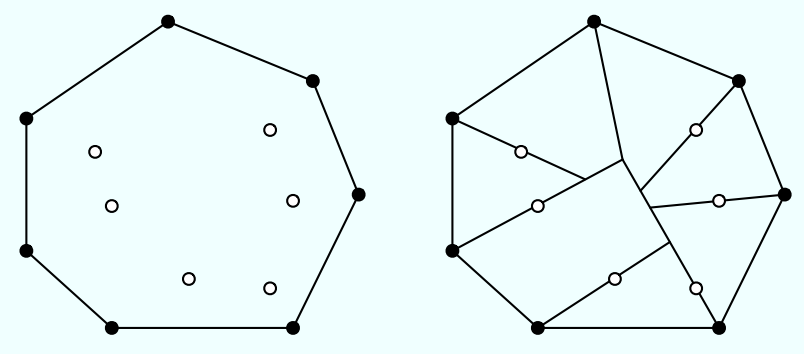

Fig. 3. The Decomposition Lemma.

We conclude this paper showing how to construct blue and red point sets with $n=i+k$ and $m=k-2+2 i$ elements respectively, such that any blue polygon contains exactly $n-2$ red points. Observe that when $k=3, R$ has exactly $m=2 n-5$ elements, and thus any blue polygonization contains exactly $\frac{m+1}{2}$ red points in its interior and $\frac{m-1}{2}$ points in its exterior.

\section{The Decomposition Lemma}

Let $P$ be a convex polygon with $n$ vertices. We call a set of convex polygons $\left\{P_{1}, \ldots, P_{n}\right\}$ with disjoint interiors a convex decomposition of $P$ if it satisfies the following conditions:

$-P_{1} \cup \ldots \cup P_{n}=P$

- Each $P_{i}$ has exactly one edge of $P$ on its boundary, called the lid of $P_{i}$.

$P_{1}, \ldots, P_{n}$ will be called the pockets of the decomposition. See Figure 3 . In the rest of this paper, all point sets or unions of point sets will be assumed to be in general position. If the vertices of a polygon $P$ are labelled $p_{1}, \ldots, p_{n}$ in the counter-clockwise order along its boundary, we might refer to $P$ as to the polygon $p_{1} p_{2} \ldots p_{n} p_{1}$. In this section we prove:

Theorem 1. [Decomposition lemma] Let $P$ be a convex polygon with $n$ vertices $p_{1}, \ldots, p_{n}$ and $S$ a set of $m$ points contained in the interior of $P, m \leq n-1$. Then there is a convex decomposition $\left\{P_{1}, \ldots, P_{n}\right\}$ of $P$ such that all the points of $S$ lie on the boundaries of $P_{1}, \ldots, P_{n}$, see Figure 3 . 
We present some preliminary results that will be useful to prove Theorem 1 .

Lemma 1. Let $T$ be a triangle with vertices $p_{1}, p_{2}$ and $p_{3}$, that contains in its interior a set $S$ of $2+x_{1}+x_{2}$ points, where $x_{1}$ and $x_{2}$ are any non-negative integers. Then, there is a point $t$ in the interior of $T$ such that one of the following situations happens:

(a) The union of the segments $t p_{1}, t p_{2}$ and $t p_{3}$ covers exactly two points from $S$, and there are $x_{1}$ and $x_{2}$ points from $S$ in the interior of the triangles $t p_{1} p_{2}$ and $t p_{2} p_{3}$, respectively.

(b) Each one of the segments $t p_{1}, t p_{2}$ and $t p_{3}$ covers exactly one point from $S$, and there are $x_{1}-1$ and $x_{2}$ points from $S$ in the interior of the triangles $t p_{1} p_{2}$ and $t p_{2} p_{3}$, respectively.

(c) Each one of the segments $t p_{1}, t p_{2}$ and $t p_{3}$ covers exactly one point from $S$, and there are $x_{1}$ and $x_{2}-1$ points from $S$ in the interior of the triangles $t p_{1} p_{2}$ and $t p_{2} p_{3}$, respectively.

Proof. Let $t_{0}$ be a point on the segment $p_{1} p_{3}$ such that the triangles $t_{0} p_{1} p_{2}$ and $t_{0} p_{2} p_{3}$ have, respectively, $x_{1}+1$ and $x_{2}+1$ points from $S$ in their interior (Figure 4 , left).
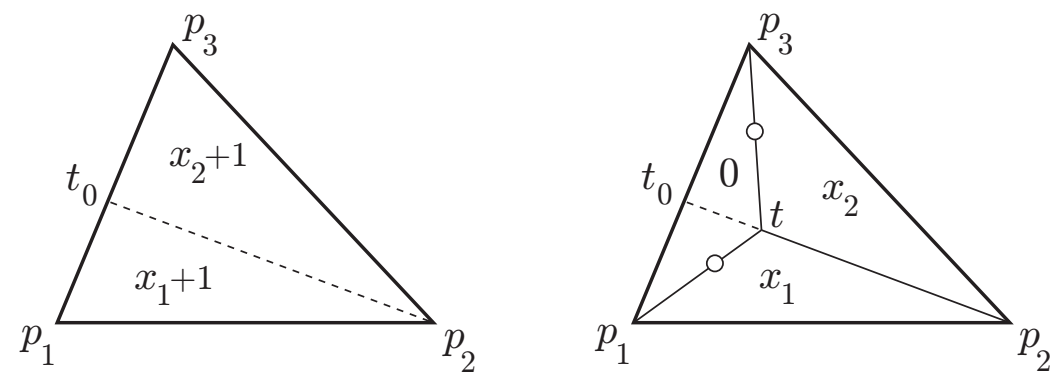

Fig. 4. Decomposing a triangle: first step.

We consider a point $t$ that moves along the segment $t_{0} p_{2}$, with initial position $t=t_{0}$, until some point from $S$ is encountered by one or both of the segments $t p_{1}$ and $t p_{3}$.

If two points are simultaneously found, one by $t p_{1}$ and the other by $t p_{3}$ we are in situation (a). Our result follows, see Figure 4, right.

Suppose then that a point $q_{1}$ from $S$ is intersected by the segment $p_{1} t$ (the case $q_{1} \in$ $p_{3} t$ is identical). Let $t_{1}$ be the intersection point of the lines generated by $p_{1} t$ and $p_{2} p_{3}$ (Figure 5, left); now we move the point $t$ towards $t_{1}$ along the line segment $p_{1} t_{1}$. If one point from $S$ is met by either of $t p_{3}$ or $t p_{2}$ we are again in case (a) and we are done (Figure 5 , center). If two points are simultaneously met by $t p_{3}$ and $t p_{2}$, we are in situation (c) (Figure 5, right).
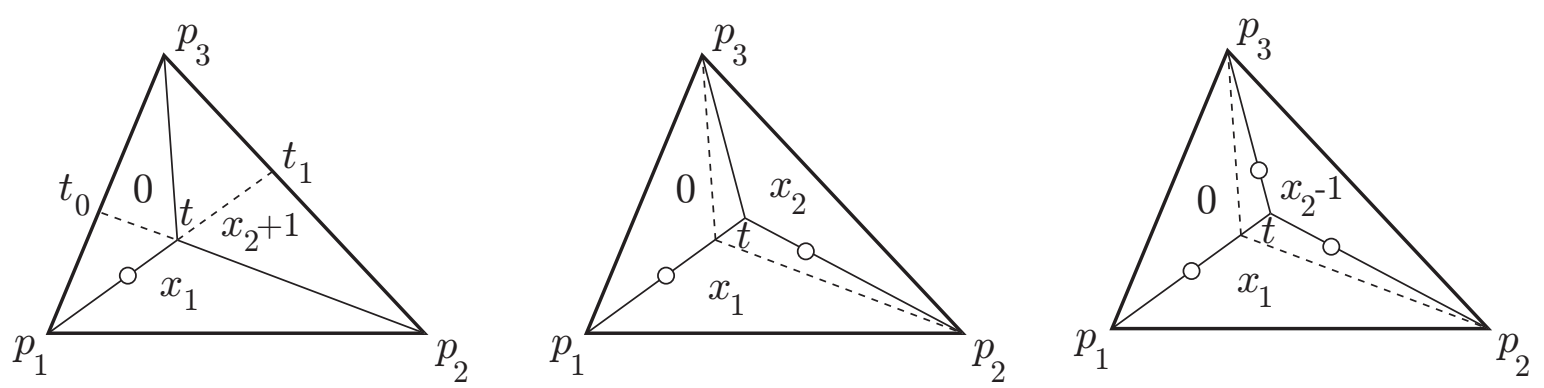

Fig. 5. Decomposing a triangle: second step. 


\section{Proof of Theorem 1}

Observe that we can assume that $m=n-1$, for otherwise we can add $(n-1)-m$ dummy points to the set $S$, obtain a convex partition, and then remove the dummy points.

We prove our result by induction on $n$; the base case $n=3$ follows from Lemma 1 with $x_{1}=x_{2}=0$. Suppose that the vertices of $P$ are labeled $p_{1}, \ldots, p_{n}$ in the counter-clockise direction along its boundary such that the lower vertex of $P$ is precisely $p_{n}$, and $p_{n}$ lies on the origin, (refer to Figure 6). Every point $q$ can be described by its polar coordinates $(r(q), \varphi(q))$, where $r(q)$ is the distance from $q$ to the origin and $\varphi(q)$ is the angle from the positive axis $+x$ to the ray through $q$ with apex at the origin.

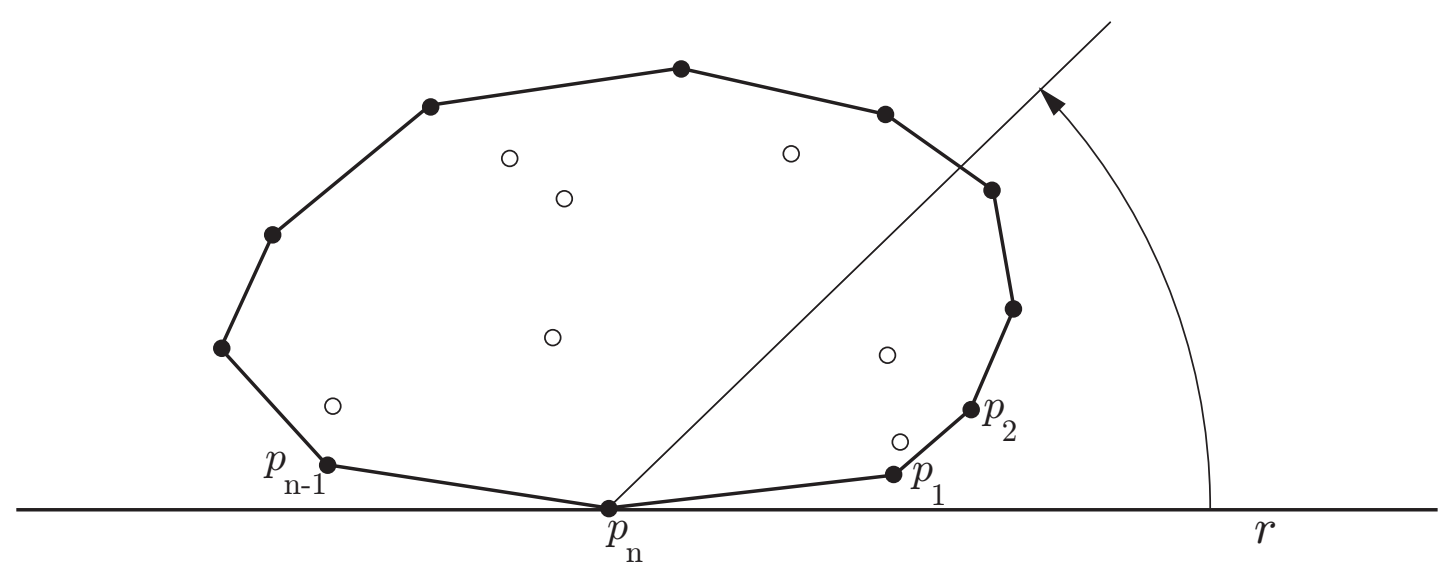

Fig. 6. Choice of the reference.

For every value $\alpha$ in the interval $[0, \pi]$ we define a function $g$ as follows:

$$
g(\alpha)=|\{q \in S \mid \varphi(q)<\alpha\}|-\left|\left\{p_{i} \mid \varphi\left(p_{i}\right)<\alpha, 1 \leq i<n\right\}\right| .
$$

Therefore, in particular,

$$
g\left(\varphi\left(p_{1}\right)\right)=0-0=0
$$

$g\left(\varphi\left(p_{n-1}\right)\right)=(n-1)-(n-2)=1$;

$g\left(\varphi\left(p_{i}\right)\right)=\mid\left\{q \in S \mid q\right.$ is in the interior of the polygon with vertices $\left.p_{n}, p_{1}, \ldots, p_{i}\right\} \mid-(i-1)$.

Let $j$ be the smallest index such that $j>1$ and $g\left(\varphi\left(p_{j}\right)\right) \geq 0$; such an index must exist because $g\left(\varphi\left(p_{n-1}\right)\right)=1$. Several cases arise.

Case 1: $g\left(\varphi\left(p_{j}\right)\right)=0$. In this case the number of points from $S$ inside the polygon $p_{n} p_{1} \ldots p_{j} p_{n}$ is exactly $j-1$ and we have $g\left(\varphi\left(p_{i}\right)\right)<0$ for all the values of $i$ such that $1<i<j$, see Figure 7 .

The polygon $\widehat{P}=p_{n} p_{j} p_{j+1} \ldots p_{n-1} p_{n}$ has $n-j+1$ vertices; let $\widehat{S}$ be the set of points of $S$ that are interior to $\widehat{P}$. Since $|\widehat{S}|=(n-1)-(j-1)=n-j<n-1$, we can apply induction to the polygon $\widehat{P}$ and the point set $\widehat{S}$ and obtain a convex partitioning $\Pi$ of $\widehat{P}$; let $Q \in \Pi$ be the pocket of $\widehat{P}$ whose lid is $p_{n} p_{j}$. Let $Q_{j-1}$ be the convex polygon obtained by the union of polygons $Q$ and $p_{n} p_{1} p_{2} \ldots p_{j} p_{n}$; note that $Q_{j-1}$ contains exactly $j-1$ points from $S$, namely the set $S_{j-1}=S \backslash \widehat{S}$. 


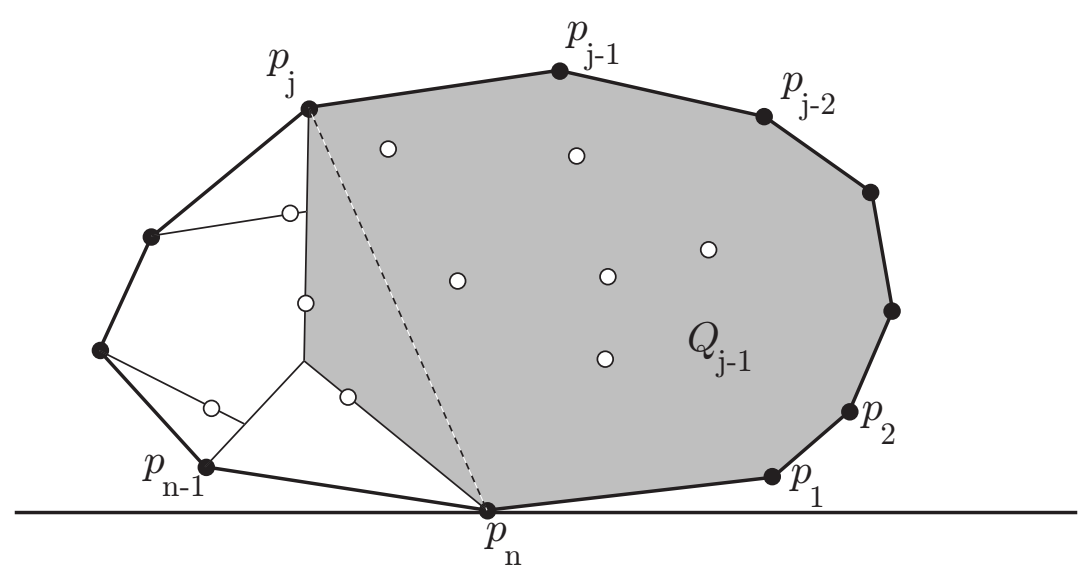

Fig. 7. First step in Case 1.

Let us consider a moving point $t$ that travels counterclockwise on the boundary of $Q_{j-1}$, starting at $p_{j}$. Before $t$ reaches $p_{n}$ some point $q \in S$ must be met by the segment $p_{j-1} t$, otherwise we would have $j-1$ points of $S$ inside $p_{n} p_{1} \ldots p_{j-1} p_{n}$ and then $g\left(\varphi\left(p_{j-1}\right)\right)>0$, contradicting the choice of $j$.

We add the chord of $Q_{j-1}$ through $p_{j-1}$ and $q$ to the decomposition of $P$ that we are constructing and remove from $Q_{j-1}$ the region swept by $p_{j-1} t$ until $q$ was found; in this way we obtain a new convex polygon $Q_{j-2}$ that contains exactly $j-2$ points from $S$ in its interior, namely the set $S_{j-2}=S_{j-1} \backslash\{q\}$ (Figure 8, left ). We repeat the preceding construction by sweeping with a chord of $Q_{j-2}$ having one endpoint anchored at $p_{j-2}$ and so on, until the claimed decomposition of $P$ is completed (Figure 8 , right).
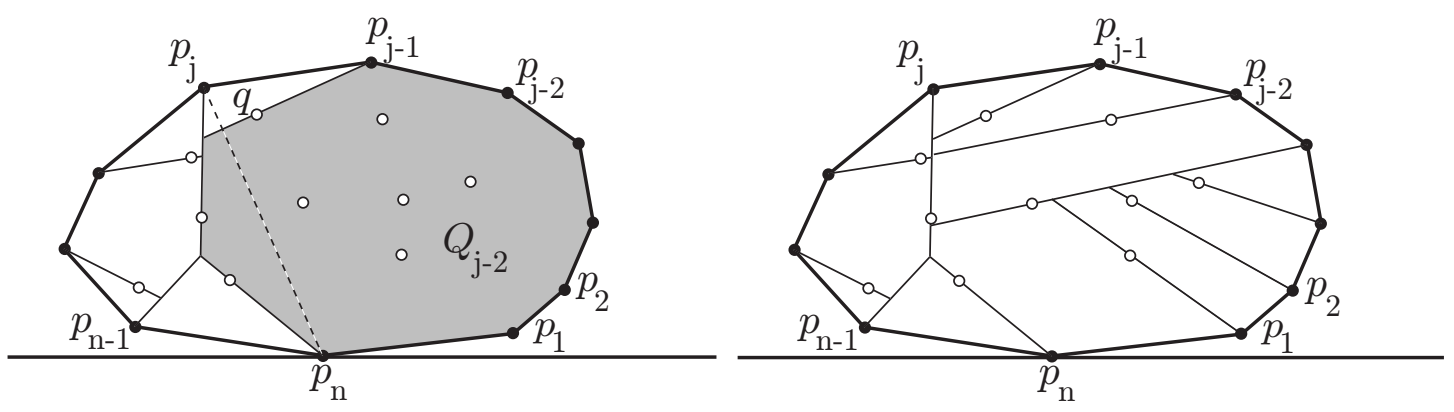

Fig. 8. Iterative step and final construction for Case 1.

Case 2: $g\left(\varphi\left(p_{j}\right)\right)>0$. In this case the number of points from $S$ inside the polygon $p_{n} p_{1} \ldots p_{j} p_{n}$ is at least $j$ and we have $g\left(\varphi\left(p_{i}\right)\right)<0$ for all the values of $i$ such that $1<i<j$.

Let $y_{1}$ and $y_{2}$ be the number of points of $S$ in the interior of the polygons $p_{n} p_{1} p_{2} \ldots p_{j-1} p_{n}$ and $p_{n} p_{j} p_{j+1} \ldots p_{n-1} p_{n}$, respectively. From the preceding observations we see that $y_{1} \leq$ $j-2$ and that $(n-1)-y_{2} \geq j$; i.e., $y_{2} \leq n-1-j$.

If we define the numbers

$$
x_{1}=j-2-y_{1}, x_{2}=n-1-j-y_{2},
$$


we see that the number $x$ of points from $S$ interior to the triangle $p_{n} p_{j-1} p_{j}$ is

$$
x=(n-1)-\left(y_{1}+y_{2}\right)=(n-1)+\left(x_{1}-j+2\right)+\left(x_{2}-n+1+j\right)=x_{1}+x_{2}+2 \geq 2 .
$$

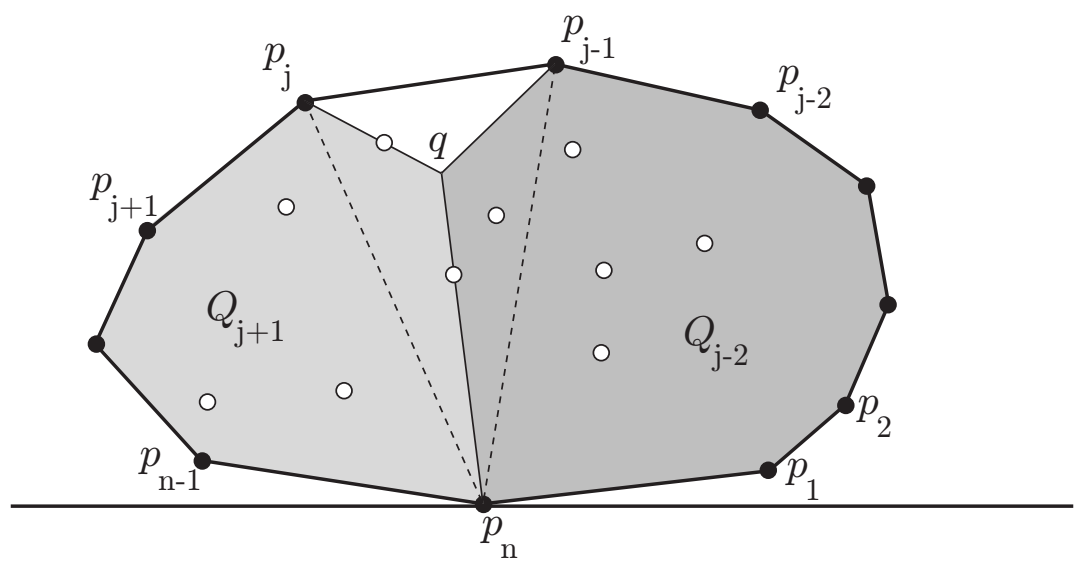

Fig. 9. First step for Case 2.

Therefore, we can apply Lemma 1 to the triangle with the numbers $x_{1}$ and $x_{2}$ associated to the sides $p_{n} p_{j-1}$ and $p_{n} p_{j}$, respectively. Let $q$ be the point such that the segments $q p_{n}$, $q p_{j-1}$ and $q p_{j}$ splits triangle $p_{n} p_{j-1} p_{j}$ as in Lemma 1.

Let $Q_{j-2}$ be the convex polygon obtained as union of the triangle $p_{n} p_{j-1} q$ and the polygon $p_{n} p_{1} p_{2} \ldots p_{j-1} p_{n}$; let $Q_{j+1}$ be the convex polygon obtained as union of the triangle $p_{n} q p_{j}$ and the polygon $p_{n} p_{j} p_{j+1} \ldots p_{n-1} p_{n}$ (Figure 9 ). Three subcases arise, that we describe separately; in all of them the segments $q p_{n}, q p_{j-1}$ and $q p_{j}$ are used for the decomposition of $P$.

Subcase 2.1: The union of the segments $q p_{n}, q p_{j-1}$ and $q p_{j}$ covers two points from $S$ and triangles $q p_{n} p_{j-1}$ and $q p_{j} p_{n}$ contain $x_{1}$ and $x_{2}$ points from $S$, respectively, in their interior.

Let $S_{j-2}$ and $S_{j+1}$ be the set of points interior to $Q_{j-2}$ and $Q_{j+1}$, respectively. We have $\left|S_{j-2}\right|=x_{1}+y_{1}=j-2$ and $\left|S_{j+1}\right|=x_{2}+y_{2}=n-1-j$ (Figure 10, left).
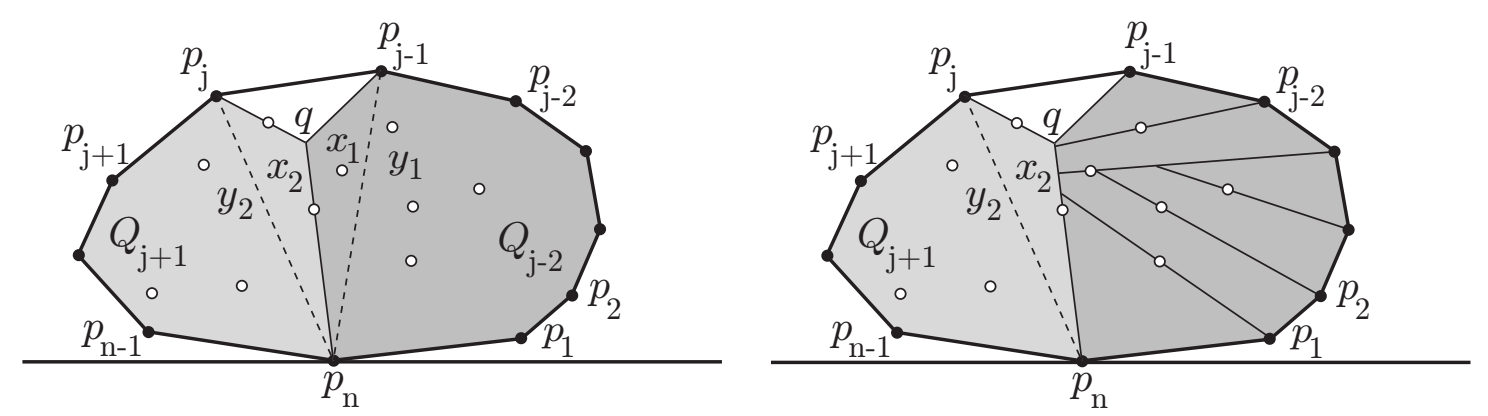

Fig. 10. Initial situation in Subcase 2.1 and decomposition of $Q_{j-2}$.

For the decomposition of $Q_{j-2}$ we consider a segment with one endpoint at $p_{j-2}$ and the other one at a moving point $t$ that travels counterclockwise on the boundary of $Q_{j-2}$, 
with starting position $t=p_{j-1}$; a point from $S$ must be found by the sweeping chord before $t$ reaches $p_{n}$, and we proceed as in the proof of Case 1 achieving the decomposition of $Q_{j-2}$ (Figure 10, right).

For the decomposition of $Q_{j+1}$ we consider a segment with one endpoint at $p_{j+1}$ and the other one at a moving point $t$ that travels clockwise on the boundary of $Q_{j+1}$, with starting position $t=p_{j}$. If some point of $S_{j+1}$ is found before $t$ reaches $p_{n}$, we cut off the swept area and iterate as in the previous situation; if this keeps happening we arrive at the decomposition of Figure 11, left.
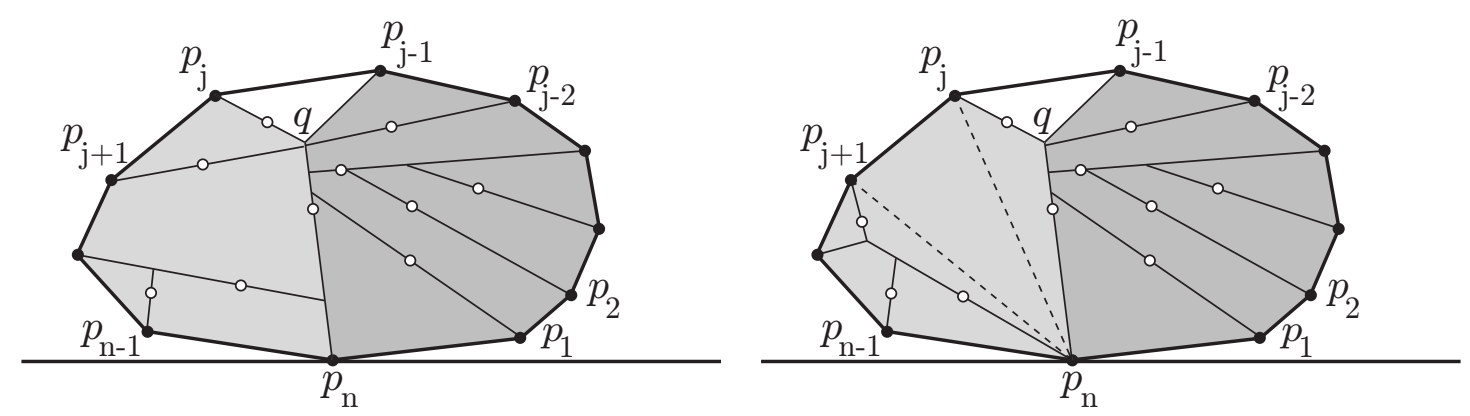

Fig. 11. Decomposition of $Q_{j+1}$ in Subcase 2.1.

If $t$ reaches $p_{n}$ and no point from $S_{j+1}$ has been encountered, all points from $S_{j+1}$ must lie in the interior of the polygon $\widehat{P}=p_{n} p_{j+1} p_{j+2} \ldots p_{n-1} p_{n}$; this polygon has $n-j=$ $\left|S_{j+1}\right|+1$ vertices and hence we can apply induction to $\widehat{P}$ and $S_{j+1}$. In the final step, we obtain the overall decomposition of $P$ by considering the pocket (with lid the edge $\left.p_{j} p_{j+1}\right)$ formed by the union of the triangles $p_{n} q p_{j}$ and $p_{j} p_{j+1} p_{n}$, together with the pocket corresponding to $p_{n} p_{j+1}$ in the decomposition of $\widehat{P}$ (Figure 11, right).
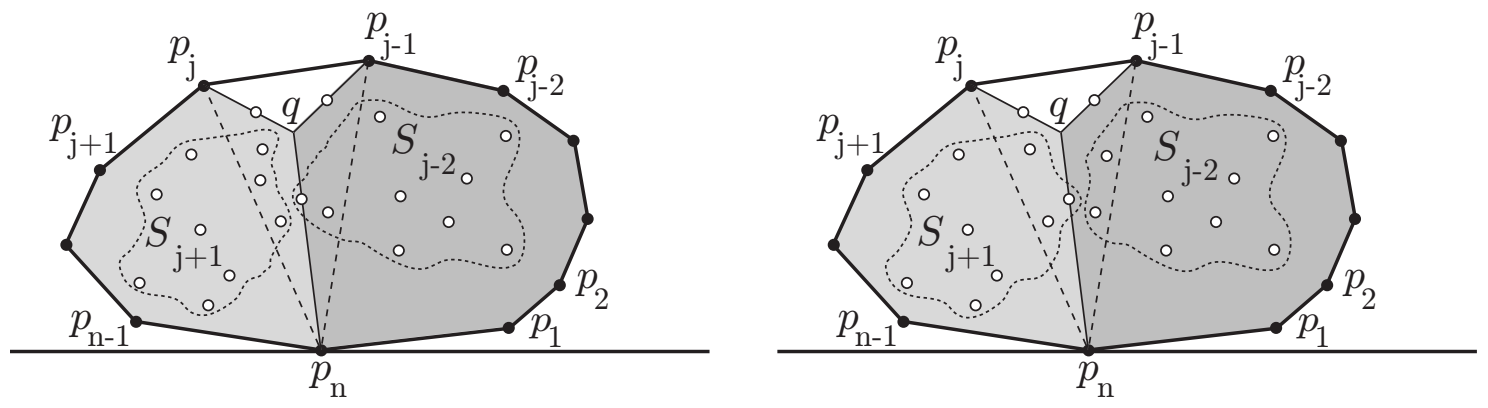

Fig. 12. Subcases 2.2 and 2.3 .

Subcase 2.2: Each one of the segments $q p_{n}, q p_{j-1}$ and $q p_{j}$ covers one point from $S$ and the interior of the triangles $q p_{n} p_{j-1}$ and $q p_{j} p_{n}$ contain, respectively, $x_{1}-1$ and $x_{2}$ points from $S$.

Let $S_{j-2}$ be the set of points of $S$ interior to $Q_{j-2}$ together with the point from $S$ covered by the segment $q p_{n}$, and let $S_{j+1}$ be the set of points of $S$ interior to $Q_{j+1}$ (Figure 12, left). We again have $\left|S_{j-2}\right|=\left(x_{1}-1\right)+y_{1}+1=j-2$ and $\left|S_{j+1}\right|=x_{2}+y_{2}=n-1-j$ and continue with the sets $S_{j-2}$ and $S_{j+1}$ as in Subcase 2.1 . 
Subcase 2.3: Each one of the segments $q p_{n}, q p_{j-1}$ and $q p_{j}$ covers one point from $S$ and the interior of the triangles $q p_{n} p_{j-1}$ and $q p_{j} p_{n}$ contain, respectively, $x_{1}$ and $x_{2}-1$ points from $S$.

Let $S_{j-2}$ be the set of points of $S$ interior to $Q_{j-2}$ and let $S_{j+1}$ be the set of points of $S$ interior to $Q_{j+1}$ together with the point from $S$ covered by the segment $q p_{n}$ (Figure 12, right). We again have $\left|S_{j-2}\right|=x_{1}+y_{1}=j-2$ and $\left|S_{j+1}\right|=\left(x_{2}-1\right)+y_{2}+1=n-1-j$ and continue with the sets $S_{j-2}$ and $S_{j+1}$ as in Subcase 2.1 .

\section{Main Result}

We recall an observation made in [5].

Observation 1 Let $P$ be a convex polygon, $p_{i} p_{j}$ an edge of $P$, and $S$ a set of points in the interior of $P$. Then there is a simple polygonal starting at $p_{i}$ and ending at $p_{j}$ such that its vertex set is $\left\{p_{i}, p_{j}\right\} \cup S$, see Figure 13.
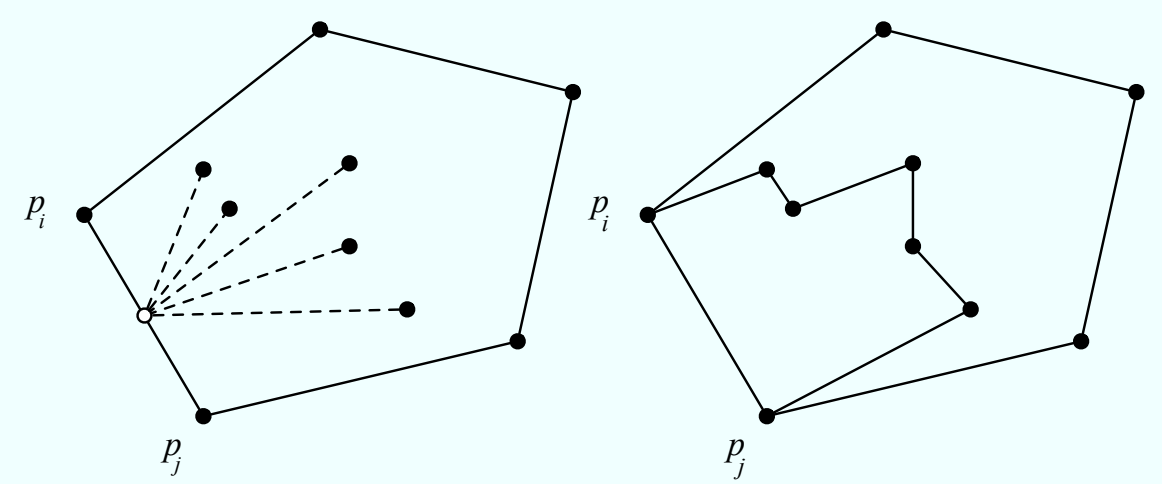

Fig. 13. The polygonal within a pocket.

We can now prove:

Theorem 2. Let $R$ and $B$ be two point sets such that $S=R \cup B$ is in general position and $R$ is contained in the interior of Conv $(S)$. Then, if the number of vertices of Conv $(S)$ is $k$ and $|R|<k$, there is a blue polygon enclosing all points of $R$. This result is tight.

Proof. The fact that $|R|$ is less than $k$ follows from the example depicted in Figure 2. By Theorem 1, we can obtain a convex decomposition $\left\{P_{1}, \ldots, P_{k}\right\}$ of $\operatorname{Conv}(B)$ such that all the points in $R$ belong to the boundaries of $P_{1}, \ldots, P_{k}$. By the previous observation, in each $P_{i}$ we can find a polygonal contained in $P_{i}$, that starts and ends at the vertices of $P_{i}$ in $\operatorname{Conv}(B)$ and contains only all the elements of $B$ in the interior of $P_{i}$. Concatenate the polygonal chains thus obtained. It is clear that this way we obtain a blue polygon that encloses all the elements of $R$, see Figure 14 .

\section{Revisiting Enclosed Point Sets and Areas}

It was proved in [5] that given $S=R \cup B$ as before, there always exists a blue polygonization that encloses at least half of the elements of $R$, and that this value is essentially 

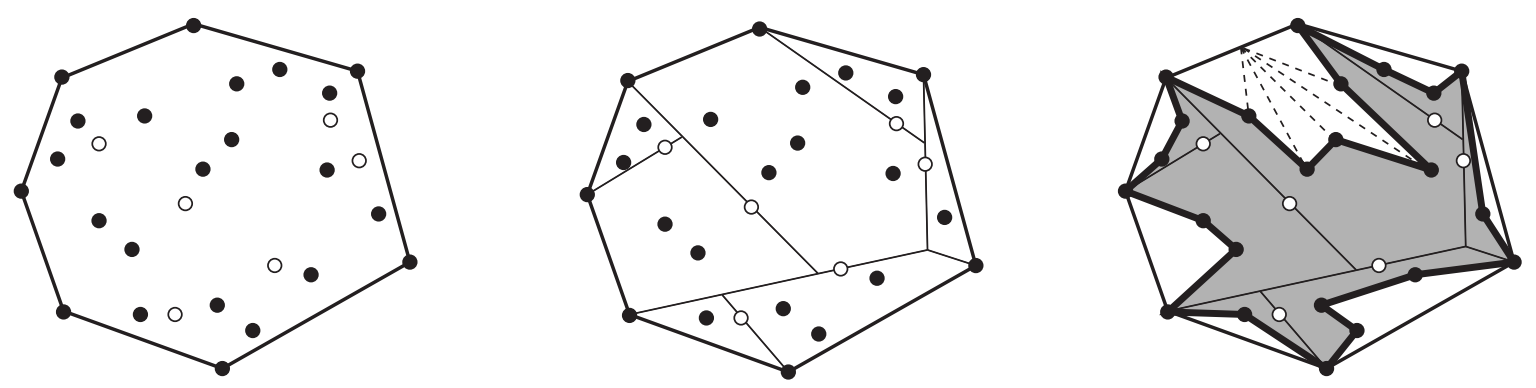

Fig. 14. Construction of the enclosing polygonization.

tight. The proof of the latter fact combined an argument on the sums of the external and internal angles of a simple polygon, and the placement of an extremely large set of red points, evenly spaced on infinitesimally small circles centered at the blue points (those outside $\operatorname{Conv}(B)$ are discarded). This gives a construction in which any blue polygonization would enclose almost half of the elements of $R$. We provide next a new proof of this result which is simpler and yields tight bounds.

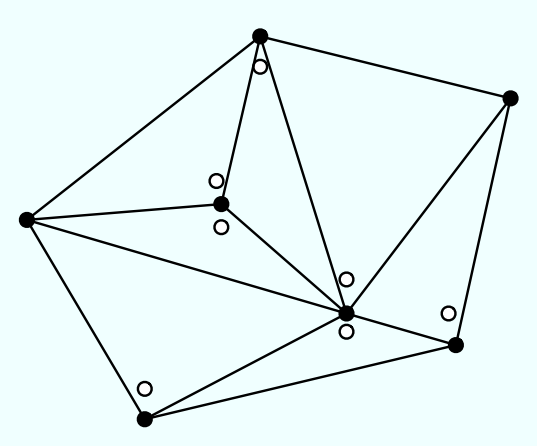

Fig. 15. The points represented by the empty circles cover the triangles with solid black point.

We recall that $k$ and $i$ denote the number of elements of $B$ on the boundary of $\operatorname{Conv}(B)$, and the number of elements of $B$ in the interior of $\operatorname{Conv}(B)$.

Given a set $B$ of blue points, we say that a set of red points $R$ covers all the triangles of $B$ if any triangle determined by three elements of $B$ contains at least one element of $R$ in its interior. We recall the following result proved first by Katchalsky [12], and independently a few years later by Czyzowicz et al [6].

Theorem 3. Let $B$ be a set of blue points. Then there exists a set $R$ consisting of $k+2 i-2$ red points in the interior of $\operatorname{Conv}(B)$ that covers all the triangles of $B$, the bound is tight. See Figure 15.

Since any triangulation of $B$ consists of $k-2+2 i$ triangles, it also follows from the same observation that any triangle with vertices in $B$, containing no element of $B$ in its interior, contains exactly one element of $R$ in its interior. If any such triangle $t$ had two or more elements of $R$, any triangulation of $B$ having $t$ as one of its elements, would include some triangle empty of elements from $R$. Since any triangulation of a polygon with $n$ vertices contains exactly $n-2$ triangles we get: 
Lemma 2. Let $B$ be a point set with $n=k+i$ elements. Then if $R$ has $m=k-2+2 i$ elements and covers all the triangles of $B$, any blue polygonization encloses $n-2=k+i-2$ elements of $R$ in its interior and leaves $i$ elements of $R$ in the exterior.

When we have $k=3$ in the situation of the preceding Lemma (i.e., when the convex hull of $B$ is a triangle), $R$ has $m=2 n-5$ elements, and thus any blue polygonization encloses $n-2=\frac{m+1}{2}$ elements of $R$ exactly. Therefore taking these sets $R$ and $B$ we arrive to the following result:

Theorem 4. There are sets of points $B$ and $R$ with $n$ and $m$ points respectively, $R \subset$ Conv $(B)$, such that any blue polygonization encloses $\frac{m+1}{2}$ elements of $R$.

In [5] it was also proved that given any point set $S$ in general position there always exist two polygonizations of $S$ such that the union of the regions they enclose is the whole interior of the convex hull of $S$. Therefore one of them contains at least half of the area of $\operatorname{Conv}(S)$. How tight is this bound? Inspired by the techniques in [9], we conclude this work observing that by using Pick's Theorem and allowing collinearities we can show that the mentioned bound, up to a constant, is tight. We state first Pick's theorem; recall that a point with integer coordinates is called a lattice point.

Theorem 5. Lat $P$ be a simple polygon such that its vertices are lattice points. Let $i(P)$ and $b(P)$ be the number of lattice points in the interior and on the boundary of $P$ respectively, and let $A(P)$ be the area enclosed by $P$. Then:

$$
A(P)=\frac{1}{2} b(P)+i(P)-1 .
$$

Now notice that if $i(p)=0$ then the area of $P$ is $\frac{1}{2} b(P)-1$. Let $L(n)$ be the set of lattice points $p=(i, j)$ such that $1 \leq i, j \leq n$. Then $L(n)$ contains $n^{2}$ lattice points, and the area of the convex hull of $L(n)$ is $(n-1)^{2}$. It now follows by Pick's Theorem that any polygonization of $L(n)$ has area $\frac{n^{2}}{2}-1$. As $n$ grows, the area of any polygonization of $L(n)$ approaches half of the area of $\operatorname{Conv}(L(n))$.

\section{References}

1. M. Ajtai, V. Chvátal, M. M. Newborn, and E. Szemerédi, Crossing-free subgraphs, Annals Discrete Math. 12 (1982), 9-12.

2. S.G. Akl, A lower bound on the maximum number of crossing-free Hamiltonian cycles in a rectilinear drawing of $K_{n}$, Ars Combinatoria, volume 7, 1979, pages 7-18.

3. P. Brass, W. Moser and J. Pach, Research problems in discrete geometry, Springer-Verlag, New York, 2005.

4. H.S.M. Coxeter, Introduction to geometry, Wiley, New York, 1969.

5. J. Czyzowicz, F. Hurtado, J. Urrutia, and N. Zaguia, On polygons enclosing point sets, Geombinatorics, Vol. XI-1, pp. 21-28, 2001.

6. J. Czyzowicz, E. Kranakis, and J. Urrutia, Guarding the Convex Subsets of a Point Set. 12th Canadian Conference on Computational Geometry, August 16-19th 2000, Fredericton, New Brunswick, Canada, 47-50. 
7. O. Devillers, F. Hurtado, G. Károlyi and C. Seara, Chromatic variants of the Erdös-Szekeres Theorem, Computational Geometry: Theory and Applications, 26(3), 2003, 193-208.

8. A. Dumitrescu and J. Pach, Partitioning colored point sets into monochromatic parts, International Journal of Computational Geometry and Applications, 12(5), 2002, 401-412.

9. S. Fekete, On simple polygonalizations with optimal area, Discrete and Computational Geometry, Volume 23 - Number 1, pp. 73-110, 2000.

10. E. Friedman, 30 Two-Colored Points with No Empty Monochromatic Convex Fourgons, Geombinatorics, Volume XIV, 2 (2004) 53-54.

11. M.R. Garey and David S. Johnson (1979). Computers and Intractability: A Guide to the Theory of NP-Completeness. W.H. Freeman, pp.211212.

12. M. Katchalski, A, Meir, On empty triangles determined by points in the plane. Acta Math. Hungar. 51, 323-328 (1988).

13. M. Newborn and W. O. J. Moser, Optimal crossing-free Hamiltonian circuit drawings of the $K^{n}$, J. Combinat. Theory, Ser. B 29 (1980), 13-26.

14. G. Pick, Geometrisches zur Zahlenlehre, Zeitschrift des Vereins, "Lotos", Prague, 1899, 311319.

15. M. Sharir, nd E. Welzl, On the Number of Crossing-Free Matchings, Cycles, and Partitions, SIAM J. Comput. in press.

Received: July, 2008

Final version received: July, 2008 